\title{
Determination of Genetic Components through Triple Test Crosses in Okra [Abelmoschus esculentus (L) Moench]
}

\author{
K. Mallikarjun*, E. Ganagappa, L. Vijaya Kumar, T. Basavaraja and S. Ramesh \\ Department of Genetics and Plant Breeding, UAS, GKVK, Bengaluru, Karnataka, India \\ *Corresponding author
}

\begin{abstract}
A B S T R A C T
The triple test cross analysis in brought out that significant epistasis is present for

Keywords

Gene action, most of the characters in three crosses except primary branches in C-I; days to flowering and number of ridges in C-II; days to 50 per cent flowering and primary branches in C-III. Significant additive $\times$ additive epistasis was recorded for fruits TTC, Okra.

\section{Article Info}

Accepted:

21 July 2017

Available Online:

10 September 2017 per plan, plant height and fruit yield in all the three crosses. while ' $\mathrm{j}$ ' (additive $\times$ dominant) and ' 1 '(dominant $\times$ dominant) type epistasis found to be significant for fruit weight, fruit length, fruit diameter, fruits per plant, plant height, nodes per plant and fruit yield in across the crosses. The $\mathrm{D}$ and $\mathrm{H}$ component were significant for most of the traits in all the three crosses. the $(\mathrm{H} / \mathrm{D})^{1 / 2}$ except days to 50 per cent flowering, where as it appears significance only for fruit length, fruits per plant and plant height in C-I; fruit weight, estimates was less than unit for most of the traits.
\end{abstract}

\section{Introduction}

The success in the selection of plant breeding programme largely depends upon the availability of reliable information about the nature and magnitude of gene action present in the material being handled by the breeder. Several biometrical methods are available for obtaining information on the nature of genetic variation. The North Carolina Design III (NCD III) of Comstock and Robinson (1952) in which homozygous parents are crossed and $\mathrm{F}_{2}$ plants are back crosses to each of the parents provides reliable estimate of additive and dominance components, but it assumes to the no epistasis. Kearsey and Jinks (1968) extended this concept and included back Crosse to the F1 generation.
This type of experiment was called Triple Test Cross by Jinks et al., (1969) and it is modified by many other workers (Jinks and Perkins, 1970; Perkins and Jinks, 1971; and Jinks and Virk, 1977). This method is the most efficient for detection and estimation of epistatic variation. If epistasis is present in the material investigated, one can obtain better estimates of additive and dominance components by this method as compared to other methods (Chahal and Singh, 1974). It also provides unbiased estimates of genetic variation. Keeping in mind the above mentioned advantages of triple test cross over other methods, the present investigation was under taken in bhendi to detect the presence 
of epistasis and to estimates the additive and dominance components of genetic variation in three crosses of okra for eleven quantitative characters.

\section{Materials and Methods}

The parents, $\mathrm{F}_{1} \mathrm{~S}$ and $\mathrm{F}_{2} \mathrm{~S}$ of the hybrid combination viz., Pusa Sawani $\times$ Sel-7 (C-I), DOV-1 $\times$ Sel-4(C-II) and DOV-1 $\times$ Arka Anamika (C-III) were raised in kharif 2010 to effect triple test crosses. 12, 18 and 11 plants were randomly chosen from each of $F_{2}$ of the respective crosses and were back crossed as a male with their respective three tester P1, P2 and $\mathrm{F} 1(\mathrm{P} 1 \times \mathrm{P} 2)$ to obtain $\mathrm{L}_{1 \mathrm{i}}, \mathrm{L}_{2 \mathrm{i}}$ and $\mathrm{L}_{3 \mathrm{i}}$ families respectively. 36, 54 and 33 families obtained from respective crosses were raised in RCBD design during summer 2011. From each family eight randomly selected plants were taken for recording the data on eleven metric traits. The method proposed by Jinks and Perkins (1970) was applied to detect epistasis and to test and estimate the additive and dominance components of genetic variance.

Test of epistasis: For detection of epistasis, the contrast $\left(\overline{\mathrm{L}}_{1 \mathrm{i}}+\overline{\mathrm{L}}_{2 \mathrm{i}}-2 \overline{\mathrm{L}}_{3 \mathrm{i}}\right)$ of each of the three crosses was computed where, $\mathrm{L}_{1}$ and $\mathrm{L}_{2}$ are the two parents of each cross and $\mathrm{L}_{3}$ is the $\mathrm{F}_{1}$ of these parents. $\mathrm{L}_{1 \mathrm{i}}$ is the mean of the plants obtain by crossing $i^{\text {th }} \mathrm{F}_{2}$ plant (where $\mathrm{i}$ $=1$ to 12,18 and 11 in C-I, C-II and C-III, respectively), $\overline{\mathrm{L}}_{1}, \overline{\mathrm{L}}_{2 \mathrm{i}}$ is the mean of the plants obtained by crossing an $\mathrm{i}^{\text {th }} \mathrm{F}_{2}$ plant (where $\mathrm{i}=$ 1 to 12,18 and 11) of each respective cross to $\mathrm{L}_{2}$ and $\overline{\mathrm{L}}_{3 \mathrm{i}}$ is the mean of the plants obtained by crossing an $\mathrm{i}^{\text {th }} \mathrm{F}_{2}$ plant (Where $\mathrm{i}=1$ to 12 , 18 and 11) of respective cross to $L_{3}$.

After summing over the replications, to estimates sum of square due to epistasis, the sum of 12, 18 and 11 squared deviations of $\left.\overline{\mathrm{L}}_{1 \mathrm{i}}+\overline{\mathrm{L}}_{2 \mathrm{i}}-2 \overline{\mathrm{L}}_{3 \mathrm{i}}\right)$ from zero for 12, 18 and 11 degrees of freedom, in cross 1 , cross 2 and cross 3 respectively, was computed. The pooled mean sum of squares due to within progeny families for testing the significance of the epistasis was obtained by the variance within $\mathrm{L}_{1 \mathrm{i}}, \mathrm{L}_{2}, 2 \mathrm{~L}_{3 \mathrm{i}}$ families for $252\{12 \times 3$ $\times(8-1)\}, 37 \overline{8}\{18 \times 3 \times(8-1)\}$ and $231\{11 \times$ $3 \times(8-1)\}$ degrees of freedom.

The epistasis sum of squares for 12, 18 and 11 degrees of freedom was partitioned into an item for ' $i$ ' type epistasis (homozygote $\times$ homozygote) for one degree of freedom and an (j) + (l) type epistasis (homozygote $\times$ heterozygote and heterozygote $x$ heterozygote). Item for 11,17 and 10 degree of freedom in C-I, C-II and C-III respectively was computed.

\section{Results and Discussion}

In the present study, triple test cross analysis was conducted in bhendi with three crosses involving the parents of different habits. Analysis of variance to detect epistasis in the three crosses is presented in table 1. The results of the present study revealed the presence of epistasis gene action for all the characters in three crosses except primary branches in C-I; days to flowering and number of ridges in $\mathrm{C}$-II; days to 50 per cent flowering and primary branches in C-III. This indicates that one would not have obtained a clear picture about the genetic systems regulating these characters if a procedure had been used assuming no epistasis.

Further partitioning of epistasis to its component parts revealed that (i) type of epistasis (additive $\mathrm{x}$ additive) was significant for all the characters except days to 50 per cent flowering, in C-II. Whereas it appears significance only for fruit length, fruits per plant and plant height in C-I; fruit weight, fruits per plant, internodel length and fruit yield in C-III. The rest of epistatic components i.e., additive $\mathrm{x}$ dominance and 
dominance $\mathrm{x}$ dominance $(\mathrm{i}+\mathrm{j})$ type were significant for all the characters in C-I; all the characters except days to 50 per cent flowering and ridges per fruit in C-II; days to 50 per cent flowering primary branches and internodel length in C-III. These results were in agreement with those reported by Saravanan et al., (2005) and Panda \& Singh (2000) in bhendi, El-Lawendey et al., (2010) in cotton.

\section{Additive and dominance components}

The present study also indicated that the importance of additive and dominance genetic components in all the crosses for character studied (Table 2). In the absence of epistasis, analysis of variance for sums and differences provide a direct tests of the significance of additive (significance of sums) and dominance components (significance of differences). The sums item were significant for all the characters in across the crosses except fruit length and internodel length in C$\mathrm{I}$; fruit diameter in C-II; internodel length in C-III. Difference item was significant for all the characters in all the three crosses except fruit diameter, ridges per plant, plant height and internodel length in C-I; days to 50 per cent flowering in C-III.

The estimates of additive (D) and dominant (H) components in the triple test crosses were presented in (Table 3) accordingly, The estimate of additive genetic component (D) was highly significant for all characters except fruit length and internodal length in CI and fruit diameter in C-II. The etimate of dominance genetic component $(\mathrm{H})$ was significant fo days to flowering, fruit weight, fruit diameter, number of fruits, primary branches, number of nodes and fruit yield per plant in C-I. The $\mathrm{H}$ component was highly significant for all characters in C-II, while it was significant for all characters except days to flowering in C-III, while estimates of both additive and dominance genetic components were highly significant for days to flowering, fruit weight, fruit diameter, number of fruits, primary branches, number of nodes and fruit yield per plant commonly across three crosses. In all cases, additive component was greater in magnitude compared with corresponding dominance component The of degree of domimance $(\mathrm{H} / \mathrm{D})^{1 / 2}$ was less than one for all characters except fruit length and number of ridges in $\mathrm{C}$-I; number of ridges and internodal length in C-II and number of fruits, fruit diameer, number of nodes, internodel length and fruit yield per plant in C-III.

Scientific plant breeding is a stochastic process. The primary need of a plant breeder is an objective evaluation of current material to decide which course of action to take in raising the next generation. Information on the precise type of gene action involved in the inheritance of characters is in designing effective breeding strategies for efficient utilization of available germplasm in crops breeding program. The plant breeder must possess an adequate knowledge of relative magnitude of components of genetic variance (i.e., additive, dominance and epistasis) indicators of its potential various biometrical methods have been used in different crops in the past to dissect components of genotypic. In most of the designs used, it is assumed that non-allelic interactions are absent, whereas the fact is often contrary to this assumption (Mather and Jinks, 1982). The importance of epistasis in crop plants the expression of several agronomic traits has been reported in a number of instances. Most of the designs rarely provide a valid test of epistasis. Therefore a test called Triple Test Cross (Kearsey and Jinks, 1968) which is the extension of North Carolina Design III of Comstock and Robinson (1952) has been adopted in the present investigation which not only provides unambiguous test for epistasis but also provides efficient estimates of additive and dominance components of genetic variance. 
Table.1 Mean sum of squares due to epistasis for 11 quantitative characters in three crosses of okra

\begin{tabular}{|c|c|c|c|c|c|c|c|c|}
\hline Source & Cross & $\begin{array}{l}\text { Degrees of } \\
\text { freedom }\end{array}$ & $\begin{array}{l}\text { Days to } 50 \text { per } \\
\text { cent flowering }\end{array}$ & Fruit weight (g) & $\begin{array}{l}\text { Fruit length } \\
\quad(\mathrm{cm})\end{array}$ & $\begin{array}{l}\text { Number of } \\
\text { ridges }\end{array}$ & $\begin{array}{c}\text { Fruit } \\
\text { diameter } \\
(\mathbf{c m})\end{array}$ & $\begin{array}{l}\text { Number of } \\
\text { fruits }\end{array}$ \\
\hline $\begin{array}{l}\text { Complete epistasis } \\
\left(\bar{L}_{1 \mathrm{i}}+\overline{\mathrm{L}}_{2 \mathrm{i}}-2 \mathrm{~L}_{3 \mathrm{i}}\right)\end{array}$ & $\begin{array}{l}\text { C-I } \\
\text { C-II } \\
\text { C-III }\end{array}$ & $\begin{array}{l}12 \\
18 \\
11\end{array}$ & $\begin{array}{c}5.93 * \\
4.76 \\
11.42 \\
\end{array}$ & $\begin{array}{l}209.35 * * \\
151.84 * * \\
126.04 * *\end{array}$ & $\begin{array}{c}20.102 * * \\
6.38 * * \\
12.42 * *\end{array}$ & $\begin{array}{c}0.74 * \\
0.89 \\
1.29 * *\end{array}$ & $\begin{array}{l}2.76 * * \\
0.29 * * \\
0.15 * *\end{array}$ & $\begin{array}{l}111.44 * * \\
261.34 * * \\
80.25\end{array}$ \\
\hline $\begin{array}{l}\text { Additive } \times \text { Additive (i type) of } \\
\text { epistasis }\end{array}$ & $\begin{array}{l}\text { C-I } \\
\text { C-II } \\
\text { C-III }\end{array}$ & $\begin{array}{l}1 \\
1 \\
1\end{array}$ & $\begin{array}{c}3.13 \\
5.51 \\
13.04 \\
\end{array}$ & $\begin{array}{c}72.85 \\
386.41 * * \\
150.00 * \\
\end{array}$ & $\begin{array}{c}76.89 * * \\
13.04 * \\
4.61\end{array}$ & $\begin{array}{c}0.82 \\
3.94^{* *} \\
0.95\end{array}$ & $\begin{array}{c}0.34 \\
0.28 * \\
0.05\end{array}$ & $\begin{array}{c}124.98 * \\
2405.44 * \\
269.06 * *\end{array}$ \\
\hline $\begin{array}{l}\text { Additive } \times \text { Dominance }+ \\
\text { Dominance } \times \text { Dominance }(j \text { and } 1 \\
\text { type }) \text { of epistasis }\end{array}$ & $\begin{array}{c}\text { C-I } \\
\text { C-II } \\
\text { C-III } \\
\end{array}$ & $\begin{array}{l}11 \\
17 \\
10 \\
\end{array}$ & $\begin{array}{c}6.19 * * \\
4.72 \\
11.26 \\
\end{array}$ & $\begin{array}{l}221.76^{* *} \\
138.05^{* *} \\
123.65^{* *}\end{array}$ & $\begin{array}{c}14.93 * * \\
5.98 * * \\
13.20 * *\end{array}$ & $\begin{array}{c}0.73 * \\
0.71 \\
1.32 *\end{array}$ & $\begin{array}{l}2.98 * * \\
0.29 * * \\
0.16 * *\end{array}$ & $\begin{array}{c}110.21 * * \\
164.85^{* *} \\
61.37 * *\end{array}$ \\
\hline Within progeny families & $\begin{array}{c}\text { C-I } \\
\text { C-II } \\
\text { C-III }\end{array}$ & $\begin{array}{l}252 \\
378 \\
231 \\
\end{array}$ & $\begin{array}{l}3.00 \\
3.52 \\
8.48\end{array}$ & $\begin{array}{l}24.81 \\
25.41 \\
34.58 \\
\end{array}$ & $\begin{array}{l}7.62 \\
2.91 \\
1.64\end{array}$ & $\begin{array}{l}0.38 \\
0.61 \\
0.71\end{array}$ & $\begin{array}{l}0.17 \\
0.07 \\
0.04\end{array}$ & $\begin{array}{l}22.09 \\
16.24 \\
20.90\end{array}$ \\
\hline
\end{tabular}

*Significance at $\mathrm{P}=0.05$

* Significance at $\mathrm{P}=0.01$

C-I: Pusa Sawani $\times$ Sel-7 C-II: DOV-1 $\times$ Sel-4 C-III: Dov-1 $\times$ Arka Anamika.

Table. 1 cont....

\begin{tabular}{|c|c|c|c|c|c|c|c|}
\hline Source & Cross & $\begin{array}{c}\text { Degrees of } \\
\text { freedom }\end{array}$ & $\begin{array}{c}\text { Plant height } \\
\text { (cm) }\end{array}$ & $\begin{array}{l}\text { Primary } \\
\text { branches }\end{array}$ & $\begin{array}{c}\text { Number of } \\
\text { nodes }\end{array}$ & $\begin{array}{l}\text { Inter nodal } \\
\text { length }(\mathrm{cm})\end{array}$ & $\begin{array}{l}\text { Fruit yield } \\
\text { plant }^{-1}(\mathrm{~g})\end{array}$ \\
\hline $\begin{array}{l}\text { Complete epistasis } \\
\left(\mathrm{L}_{1 \mathrm{i}}^{-}+\mathrm{L}_{2 \mathrm{i}}^{-}-2 \mathrm{~L}_{3 \mathrm{i}}\right)\end{array}$ & $\begin{array}{c}\text { C-I } \\
\text { C-II } \\
\text { C-III }\end{array}$ & $\begin{array}{l}12 \\
18 \\
11\end{array}$ & $\begin{array}{c}4496.92 * * \\
1572.47 * * \\
415.76 * *\end{array}$ & $\begin{array}{c}0.66 \\
2.96^{* *} \\
0.44\end{array}$ & $\begin{array}{l}152.05^{* *} \\
262.30^{* *} \\
57.69 * *\end{array}$ & $\begin{array}{c}1.562 * \\
1.93 * * \\
1.23 *\end{array}$ & $\begin{array}{l}280623.6 * * \\
241735.1 * * \\
115855.4 * *\end{array}$ \\
\hline $\begin{array}{l}\text { Additive } \times \text { Additive } \\
\text { (i type) of epistasis }\end{array}$ & $\begin{array}{c}\text { C-I } \\
\text { C-II } \\
\text { C-III }\end{array}$ & $\begin{array}{l}1 \\
1 \\
1\end{array}$ & $\begin{array}{c}11156.28 * * \\
17716.29 * * \\
1028.40 *\end{array}$ & $\begin{array}{c}0.04 \\
33.81 * * \\
0.42\end{array}$ & $\begin{array}{c}33.17 \\
2405.44 * * \\
203.96 * *\end{array}$ & $\begin{array}{c}0.01 \\
11.83 * * \\
3.47 *\end{array}$ & $\begin{array}{c}291827.20 \\
1789010.00 * * \\
620513.10^{* *}\end{array}$ \\
\hline $\begin{array}{l}\text { Additive } \times \text { Dominance }+ \\
\text { Dominance } \times \text { Dominance }(j \text { and } 1 \\
\text { type }) \text { of epistasis }\end{array}$ & $\begin{array}{c}\text { C-I } \\
\text { C-II } \\
\text { C-III }\end{array}$ & $\begin{array}{l}11 \\
17 \\
10\end{array}$ & $\begin{array}{c}3891.53 * * \\
622.84 * * \\
354.49 *\end{array}$ & $\begin{array}{c}0.71 * \\
1.14 * * \\
0.44\end{array}$ & $\begin{array}{c}162.86 * * \\
136.23 * * \\
43.07 *\end{array}$ & $\begin{array}{c}1.70^{*} \\
1.35^{*} \\
1.01\end{array}$ & $\begin{array}{c}279605.1 * * \\
150718.9 * * \\
65389.59 *\end{array}$ \\
\hline Within progeny families & $\begin{array}{c}\text { C-I } \\
\text { C-II } \\
\text { C-III }\end{array}$ & $\begin{array}{l}252 \\
378 \\
231\end{array}$ & $\begin{array}{l}422.51 \\
212.25 \\
177.60\end{array}$ & $\begin{array}{l}0.37 \\
0.28 \\
0.33\end{array}$ & $\begin{array}{l}28.47 \\
17.38 \\
19.23\end{array}$ & $\begin{array}{l}0.83 \\
0.77 \\
0.65\end{array}$ & $\begin{array}{l}21959.07 \\
19142.20 \\
33901.13\end{array}$ \\
\hline
\end{tabular}

*Significance at $\mathrm{P}=0.05 \quad * *$ Significance at $\mathrm{P}=0.01$

C-I: Pusa Sawani $\times$ Sel-7 C-II: DOV-1 $\times$ Sel-4 C-III: Dov-1 $\times$ Arka Anamika. 
Table.2 Analysis of variance for detection and estimation of additive (D) and dominance (H) components of genetic variance in three crosses of okra

\begin{tabular}{|c|c|c|c|c|c|c|c|c|}
\hline \multirow[b]{2}{*}{ Source } & \multicolumn{8}{|c|}{ Mean sum of squares } \\
\hline & Cross & $\begin{array}{c}\text { Degrees of } \\
\text { freedom }\end{array}$ & $\begin{array}{l}\text { Days to } 50 \text { per } \\
\text { cent flowering }\end{array}$ & Fruit weight (g) & $\begin{array}{c}\text { Fruit } \\
\text { length } \\
\text { (cm) }\end{array}$ & $\begin{array}{l}\text { Number of } \\
\text { ridges }\end{array}$ & $\begin{array}{c}\text { Fruit } \\
\text { diameter }\end{array}$ & $\begin{array}{c}\text { Number of } \\
\text { frits }\end{array}$ \\
\hline Sums $\left(\mathrm{L}_{1 \mathrm{i}}+\mathrm{L}_{2 \mathrm{i}}\right)-$ & $\begin{array}{c}\text { C-I } \\
\text { C-II } \\
\text { C-III } \\
\end{array}$ & $\begin{array}{l}11 \\
17 \\
10 \\
\end{array}$ & $\begin{array}{c}93.38 * * \\
8.59 * * \\
58.62 * *\end{array}$ & $\begin{array}{l}486.65 * * \\
207.58 * * \\
202.69 * *\end{array}$ & $\begin{array}{c}6.38 \\
15.50 * * \\
28.02 * *\end{array}$ & $\begin{array}{l}0.98 * * \\
1.48 * * \\
3.52 * *\end{array}$ & $\begin{array}{c}1.04 * * \\
0.58 \\
0.25 * *\end{array}$ & $\begin{array}{c}260.77 * * \\
160.77 * * \\
61.23 * *\end{array}$ \\
\hline Difference $\left(\mathrm{L}_{1 \mathrm{i}}-\mathrm{t}_{2 \mathrm{i}}\right)$ & $\begin{array}{c}\text { C-I } \\
\text { C-II } \\
\text { C-III } \\
\end{array}$ & $\begin{array}{l}11 \\
17 \\
10 \\
\end{array}$ & $\begin{array}{c}5.26 * * \\
8.36 * * \\
25.62\end{array}$ & $\begin{array}{c}399.04 * * \\
46.77 * * \\
152.22 * *\end{array}$ & $\begin{array}{c}5.35 \\
8.01 * * \\
24.22 * *\end{array}$ & $\begin{array}{c}1.32 \\
1.48 * * \\
3.22 * *\end{array}$ & $\begin{array}{l}0.57 * * \\
0.45 * * \\
0.27 * *\end{array}$ & $\begin{array}{l}71.02 * * \\
69.17 * * \\
78.72 * *\end{array}$ \\
\hline Within progeny family & $\begin{array}{c}\text { C-I } \\
\text { C-II } \\
\text { C-III }\end{array}$ & $\begin{array}{l}168 \\
252 \\
154\end{array}$ & $\begin{array}{c}3.29 \\
3.43 \\
16.66\end{array}$ & $\begin{array}{l}54.46 \\
19.69 \\
28.07\end{array}$ & $\begin{array}{l}7.67 \\
3.16 \\
2.49\end{array}$ & $\begin{array}{l}0.42 \\
0.56 \\
0.73\end{array}$ & $\begin{array}{l}0.15 \\
0.12 \\
0.05\end{array}$ & $\begin{array}{l}24.43 \\
17.79 \\
16.62\end{array}$ \\
\hline
\end{tabular}

*Significance at $\mathrm{P}=0.05 \quad$ **Significance at $\mathrm{P}=0.01$

C-I: Pusa Sawani $\times$ Sel-7 C-II: DOV-1 $\times$ Sel-4 C-III: Dov- $1 \times$ Arka Anamika.

Table. 2 cont....

\begin{tabular}{|c|c|c|c|c|c|c|c|}
\hline \multirow[b]{2}{*}{ Source } & \multicolumn{7}{|c|}{ Mean sum of squares } \\
\hline & Cross & $\begin{array}{c}\text { Degrees of } \\
\text { freedom }\end{array}$ & Plant height $(\mathrm{cm})$ & $\begin{array}{l}\text { Primary } \\
\text { branches }\end{array}$ & $\begin{array}{c}\text { Number of } \\
\text { nodes }\end{array}$ & $\begin{array}{l}\text { Internodal } \\
\text { length }(\mathrm{cm})\end{array}$ & $\begin{array}{c}\text { Fruit yield plant }^{-1} \\
(\mathrm{~g})\end{array}$ \\
\hline \multirow[t]{3}{*}{ Sums $\left(\mathrm{L}_{1 \mathrm{i}} \neq \mathrm{L}_{2 \mathrm{i}}\right)-$} & C-I & 11 & $4554.32 * *$ & $4.69 * *$ & $271.99 * *$ & 1.08 & $299445.10 * *$ \\
\hline & C-II & 17 & $2047.58 * *$ & $2.14 * *$ & $130.16^{* *}$ & $2.13 * *$ & $290821.10^{* *}$ \\
\hline & C-III & 10 & $1018.34 * *$ & $2.07 * *$ & $58.63 * *$ & 0.98 & $98745.62 * *$ \\
\hline \multirow[t]{3}{*}{ Differences $\left(\mathrm{L}_{1 \mathrm{i}}-\mathrm{E}_{2 \mathrm{i}}\right)$} & C-I & 11 & 630.06 & $2.56^{* *}$ & $124.89 * *$ & 0.97 & $177725.30 * *$ \\
\hline & C-II & 17 & $1461.75 * *$ & $1.80 * *$ & $109.96 * *$ & $2.92 * *$ & $125028.10 * *$ \\
\hline & C-III & 10 & $696.29 * *$ & $1.48 * *$ & $64.40 * *$ & $2.82 * *$ & $140929.10 * *$ \\
\hline \multirow{3}{*}{ Within progeny families } & C-I & 168 & 393.41 & 0.41 & 27.99 & 0.65 & 16883.79 \\
\hline & C-II & 252 & 302.19 & 0.45 & 20.17 & 0.88 & 18268.94 \\
\hline & C-III & 154 & 194.11 & 0.25 & 16.49 & 0.72 & 23260.76 \\
\hline
\end{tabular}

*Significance at $\mathrm{P}=0.05$

$* *$ Significance at $\mathrm{P}=0.01$

C-I: Pusa Sawani $\times$ Sel-7 C-II: DOV-1 × Sel-4 C-III: Dov- $1 \times$ Arka Anamika.. 
Table.3 Estimation of additive (D) and dominance $(\mathrm{H})$ components of genotypic variance for 11 quantitative traits in three crosses of okra

\begin{tabular}{|c|c|c|c|c|c|c|c|}
\hline Source & Cross & $\begin{array}{c}\text { Days to } 50 \text { per cent } \\
\text { flowering }\end{array}$ & $\begin{array}{c}\text { Fruit weight } \\
\text { (gm) }\end{array}$ & $\begin{array}{l}\text { Fruit length } \\
(\mathrm{cm})\end{array}$ & $\begin{array}{l}\text { Number of } \\
\text { ridges }\end{array}$ & $\begin{array}{l}\text { Fruit diameter } \\
\text { (cm) }\end{array}$ & $\begin{array}{l}\text { Number of } \\
\text { fruits }\end{array}$ \\
\hline \multirow{3}{*}{$\mathrm{D}$} & C-I & $3.48 * *$ & $246.96 * *$ & 0.73 & $0.32 * *$ & $0.50 * *$ & $135.04 * *$ \\
\hline & C-II & $2.57 * *$ & $93.94 * *$ & $6.16^{* *}$ & $0.45^{* *}$ & 0.22 & $71.48 * *$ \\
\hline & C-III & $23.97 * *$ & $99.78 * *$ & $14.59 * *$ & $1.59 * *$ & $0.11^{* *}$ & $25.49 * *$ \\
\hline \multirow{3}{*}{$\mathrm{H}$} & C-I & $1.12 * *$ & $196.90 * *$ & 1.32 & 0.51 & $0.23 * *$ & $26.62 * *$ \\
\hline & C-II & $2.46^{* *}$ & $13.54 * *$ & $2.42 * *$ & $0.45^{* *}$ & $0.16^{* *}$ & $25.6^{* *}$ \\
\hline & C-III & 5.11 & $70.94 * *$ & $12.41 * *$ & $1.42 * *$ & $0.12 * *$ & $35.48 * *$ \\
\hline \multirow{3}{*}{$(\mathrm{H} / \mathrm{D})^{1 / 2}$} & C-I & 0.56 & 0.89 & 1.34 & 1.26 & 0.68 & 0.44 \\
\hline & C-II & 0.97 & 0.37 & 0.62 & 1.00 & 0.83 & 0.59 \\
\hline & C-III & 0.46 & 0.84 & 0.92 & 0.94 & 1.03 & 1.17 \\
\hline \multirow{3}{*}{$\mathrm{F}$} & C-I & 0.68 & -41.72 & -0.16 & 0.04 & 0.06 & -10.7 \\
\hline & C-II & 0.01 & $9.70 *$ & $1.45^{* *}$ & $0.04 *$ & -0.07 & -11.27 \\
\hline & C-III & -3.42 & 5.73 & -0.66 & 0.06 & 0.01 & 1.43 \\
\hline \multirow{3}{*}{$\begin{array}{c}\text { r (Sums / } \\
\text { Difference) }\end{array}$} & C-I & 0.42 & -0.41 & -0.12 & 0.18 & 0.39 & -0.34 \\
\hline & C-II & 0.02 & $0.41 *$ & $0.76^{* *}$ & $0.44^{*}$ & -0.61 & -0.45 \\
\hline & C-III & -0.81 & 0.14 & -0.11 & 0.47 & 0.01 & 0.09 \\
\hline
\end{tabular}


Table.3 cont....

\begin{tabular}{|c|c|c|c|c|c|c|}
\hline Source & Cross & $\begin{array}{c}\text { Plant height } \\
(\mathrm{cm})\end{array}$ & $\begin{array}{l}\text { Primary } \\
\text { branches }\end{array}$ & $\begin{array}{l}\text { Number of } \\
\text { nodes }\end{array}$ & $\begin{array}{c}\text { Inter nodal length } \\
(\mathrm{cm})\end{array}$ & $\begin{array}{c}\text { Fruit yield plant }^{-1} \\
\text { (g) }\end{array}$ \\
\hline $\mathrm{D}$ & $\begin{array}{l}\text { C-I } \\
\text { C-II } \\
\text { C-III }\end{array}$ & $\begin{array}{l}2377.66 * * \\
872.69 * * \\
470.99 * *\end{array}$ & $\begin{array}{l}2.44 * * \\
0.84 * * \\
1.03 * *\end{array}$ & $\begin{array}{l}139.42 * * \\
54.99 * * \\
24.08 * *\end{array}$ & $\begin{array}{c}0.24 \\
0.62 * * \\
0.15 * *\end{array}$ & $\begin{array}{c}161463.60 * * \\
136276.10 * * \\
43134.21 * *\end{array}$ \\
\hline $\mathrm{H}$ & $\begin{array}{l}\text { C-I } \\
\text { C-II } \\
\text { C-III }\end{array}$ & $\begin{array}{c}135.22 \\
579.78 * * \\
286.96 * *\end{array}$ & $\begin{array}{l}1.22 * * \\
0.67 * * \\
0.70 * *\end{array}$ & $\begin{array}{l}55.37 * * \\
44.89 * * \\
27.38 * *\end{array}$ & $\begin{array}{c}0.18 \\
1.02 * * \\
1.20 * *\end{array}$ & $\begin{array}{l}91909.46 * * \\
53379.56 * * \\
67239.04 * *\end{array}$ \\
\hline$(\mathrm{H} / \mathrm{D})^{1 / 2}$ & $\begin{array}{l}\text { C-I } \\
\text { C-II } \\
\text { C-III }\end{array}$ & $\begin{array}{l}0.23 \\
0.81 \\
0.78\end{array}$ & $\begin{array}{l}0.70 \\
0.89 \\
0.82\end{array}$ & $\begin{array}{c}0.63 \\
0.9 \\
1.06\end{array}$ & $\begin{array}{l}0.86 \\
1.27 \\
2.82\end{array}$ & $\begin{array}{l}0.75 \\
0.62 \\
1.24\end{array}$ \\
\hline $\mathrm{F}$ & $\begin{array}{l}\text { C-I } \\
\text { C-II } \\
\text { C-III }\end{array}$ & $\begin{array}{l}20.67 \\
-19.13 \\
-9.31\end{array}$ & $\begin{array}{l}-0.05 \\
0.15 \\
-0.26\end{array}$ & $\begin{array}{l}-14.96 \\
-4.25 \\
-4.09\end{array}$ & $\begin{array}{l}-0.03 \\
0.23 \\
0.05\end{array}$ & $\begin{array}{c}-12591.80 \\
3597.56 \\
-6392.60\end{array}$ \\
\hline $\begin{array}{c}\text { r (Sums / } \\
\text { Difference) }\end{array}$ & $\begin{array}{l}\text { C-I } \\
\text { C-II } \\
\text { C-III }\end{array}$ & $\begin{array}{l}0.05 \\
-0.04 \\
-0.04\end{array}$ & $\begin{array}{l}-0.07 \\
0.32 \\
-0.65\end{array}$ & $\begin{array}{l}-0.35 \\
-0.15 \\
-0.33\end{array}$ & $\begin{array}{c}-0.13 \\
0.4 \\
0.27\end{array}$ & $\begin{array}{l}-0.23 \\
0.07 \\
-0.32\end{array}$ \\
\hline
\end{tabular}

*Significance at $\mathrm{P}=0.05$ C-I: Pusa Sawani $\times$ Sel-7
**Significance at $\mathrm{P}=0.01$

C-II: DOV-1 $\times$ Sel-4 C-III: Dov-1 $\times$ Arka Anamika. 
Test and estimation of additive (D) and dominance (H) components of genotypic variance

\begin{tabular}{l|l|l|l|}
\hline Source & d.f. & MSS & EMSS \\
\hline Sums $\left(\mathrm{L}_{1 \mathrm{i}}^{-}+\mathrm{L}_{2 \mathrm{i}}\right)^{-}$ & $(\mathrm{n}-1)$ & $\mathrm{M}_{1}$ & $\sigma_{\mathrm{e}}^{2}+2 \mathrm{~m} \sigma_{\mathrm{S}}^{2}$ \\
\hline Difference $\left(\mathrm{L}_{1 \mathrm{i}}-\mathrm{L}_{2 \mathrm{i}}\right)^{-}$ & $(\mathrm{n}-1)$ & $\mathrm{M}_{2}$ & $\sigma_{\mathrm{e}}^{2}+2 \mathrm{~m}^{2}{ }_{\mathrm{D}}$ \\
\hline Within TTC progeny family & $2 \mathrm{n}(\mathrm{m}-1)$ & $\mathrm{M}_{3}$ & $\sigma_{\mathrm{e}}^{2}$ \\
Additive component $(\mathrm{D}) \sigma_{\mathrm{s}}^{2}=\frac{\mathrm{M}_{1}-\mathrm{M}_{3}}{2 \mathrm{~m}}$ & $\sigma_{\mathrm{s}}^{2}=\frac{1}{8} \mathrm{D}$ & \\
Dominance component $(\mathrm{H}) \sigma_{\mathrm{d}=\frac{\mathrm{M}_{2}-\mathrm{M}_{3}}{2 \mathrm{~m}}} \quad \sigma_{\mathrm{d}}^{2}=\frac{1}{8} H$ \\
Degree of dominance $=(\mathrm{H} / \mathrm{D})^{1 / 2}$
\end{tabular}

In addition, this design has wide applicability as it can be used to investigate both segregating and non-segregating populations arising from different generations such as $\mathrm{F}_{2}$, backcross and homozygous lines.

The result of the present study revealed the presence of digenic epistatic gene interaction in the expression of all the traits. These results emphasized the inadequacy of additive and dominance gene action to explain the inheritance of traits in a given cross, and pointed out the importance of epistasis in the inheritance of the characters. Presence of ' $i$ ' type epistatic interaction (additive $\times$ additive) suggested that selection in early segregating generation could be effective.

Though the results revealed the prevalence of both ' $i$ ' type and $(j+1)$ type of epistasis in the expression of some characters such as, fruit length, number of fruits and plant height in $\mathrm{C}$ $\mathrm{I}$; fruit weight, fruit diameter, number of fruits, plant height, primary branches, number of nodes, internodel length and fruit yield per plant in C-II and fruit weight, number of fruits, plant height, number of nodes and fruit yield per plant in C-III, the magnitude of 'i' type of epistasis was larger than that of $(j+1)$ type of epistasis for all the characters in all the three crosses. This reiterated the importance of additive $x$ additive type of interaction in the genetic control of such characters
The both additive and dominance component of genotypic variance were significant for most of the characters in all the three crosses. we have to fallow breeding methodology such has reciprocal recurrent selection for make use of both the gene action.

The degree of dominance further revealed that predominant of additive genetic component for all the characters in across the crosses. All the characters except fruit length and ridges per plant in C-I; ridges per plant and internodal length in C-II; fruit diameter, fruits per plant, nodes per plant, internodal length and fruit yield per plant in C-III, for which degree of dominance is more than unity, indicating predominant role of dominance gene action. Similar reports were made by Saravanan et al., (2005) in bhendi. Generally, from the previous results it may be concluded that the studied characters are complex inherited and controlled by additive and nonadditive gene effects. But the additive component and additive $\mathrm{x}$ additive type of epistasis were preponderant for most characters. On the other side, because of the presence of epistatic component, the selection procedure could not be fruitful in immediate progenies and process has to be delayed to later generations. Thus, recurrent selection and/or intimating population procedures may be useful in the sense that it will exploit both additive and non-additive components of genetic variation for bringing about 
improvement of such characters. Such strategy will help increase frequency of favorable alleles.

\section{References}

Chahal, G. S., and Singh, T. H. 1974. Crop Improv, 1:61-71.

Comstock, R. E., and Robinson, H. F., 1952. Estimation of average dominance of genes. In: Heterosis, Iowa State College Press, and Ames: 494-516.

Jinks, J. L., and Perkins, J. M., 1970. A general method of detecting the additive, dominance and epistatic variation. III. $\mathrm{F}_{2}$ and backcross populations. Heredity, 25: 419-423.

Jinks, J. L., and Virk, D. S., 1977. Modified triple test cross analysis to test and allow for inadequate testers. Heredity, 39: $165-170$.

Jinks, J. L., Perkins, J. M., and Breese, E. L., 1969. A general method of detecting the additive, dominance and epistatic variation for metrical traits. II. Application to inbred lines. Heredity,
24: 45-57.

Kearsy, M. J., and Jinks, J. L., 1968. A general method of detecting the additive, dominance and epistatic variation for metrical traits. I. Theory, Heredity, 23: 403-409.

Kearsy, M. J., and Jinks, J. L., 1968. A general method of detecting the additive, dominance and epistatic variation for metrical traits. I. Theory, Heredity, 23: 403-409.

Mather, K., and Jinks, J. H., 1982. Biometrical Genetics: The study of continuous variation. Chapman and Hall, London, $3^{\text {rd }}$ Edn, pp 396.

Panda, P. K., and Singh, K. P. 2000. Modified triple test cross analysis for yield and yield components in okra [Abelmoschus esculentus (L) Moench]. Indian J. Genet., 60: 569-571.

Saravanan, K., Sabesan, T., Senthil Kumar, N., Ganasan, J., 2005. Triple test cross analysis in bhendi [Abelmoschus esculentus (L) Moench]. Indian J. Agric. Res., 39: 242-248.

\section{How to cite this article:}

Mallikarjun, K., E. Ganagappa, L. Vijaya Kumar, T. Basavaraja and Ramesh, S. 2017. Determination of Genetic Components through Triple Test Crosses in Okra [Abelmoschus esculentus (L) Moench]. Int.J.Curr.Microbiol.App.Sci. 6(9): 1991-1999.

doi: https://doi.org/10.20546/ijcmas.2017.609.244 